\title{
A Note Concerning the Two-Step Lax-Wendroff Method in Three Dimensions
}

\author{
By B. Eilon
}

\begin{abstract}
The two-step Lax-Wendroff method in three spatial dimensions is discussed and, dealing with its linear stability in the hydrodynamic case, the sufficiency of the von Neumann condition is proved.
\end{abstract}

In their paper [1], Rubin and Preiser suggest a difference scheme for the conservation law:

$$
W_{t}+\partial f_{i} / \partial x_{i}=0 .
$$

Their scheme is an extension of Richtmyer's two-step method to three spatial dimensions. In order to deal with the linear stability they take the linearized equation: $W_{t}+A_{i} \cdot \partial w / \partial x_{i}$ (where $A_{i}=\partial f_{i} / \partial W$ are taken locally constant), and, in order to get a stability criterion, they compute the amplification matrix:

$$
G=I-\frac{2}{3} i \lambda\left[\operatorname{Cos} \xi_{1}+\operatorname{Cos} \xi_{2}+\operatorname{Cos} \xi_{3}\right] M-2 \lambda^{2} M^{2} .
$$

Here $\lambda=\Delta t / \Delta x_{1}=\Delta t / \Delta x_{2}=\Delta t / \Delta x_{3}, \xi_{i}=k_{i} \Delta x_{i}$ (where $k_{i}$ are the dual variables) and $M=A_{1} \operatorname{Sin} \xi_{1}+A_{2} \operatorname{Sin} \xi_{2}+A_{3} \operatorname{Sin} \xi_{3}$.

To prove sufficiency of the von Neumann condition, Rubin and Preiser use a theorem due to Kreiss [3] where the dissipativity of the scheme is assumed. However, it is easy to verify that their scheme is not dissipative because for $\xi=(\pi, 0,0)$ (so that $|\xi|=\pi$ ), for example, $M$ is the null matrix and $G=I$ so that its eigenvalues are on the unit circle, as is the case for two dimensions (see [4]).

We give a different proof for the sufficiency of the von Neumann condition but only for the hydrodynamic case. (This is an extension of Richtmyer's proof in [2] to three spatial dimensions.)

In this case, $W=(\rho, \rho u, \rho v, \rho w, E)$, where $\rho, E$ and $\mathrm{V}=(u, v, w)$ are the density, total energy per unit volume and the velocity vector, respectively. We shall make use of the following sufficiency theorem (see [2]): "If $G$ has a complete set of eigenvectors and there exists a constant $\delta$ such that $\Delta \geqq \delta>0$, where $\Delta^{2}$ is the Gram determinant of the normalized eigenvectors, then the von Neumann condition is sufficient as well as necessary for stability".

Instead of calculating the eigenvectors of $G$, we shall consider another matrix $G^{\prime}$ obtained from $G$ by a similarity transformation. We introduce $W^{\prime}=(\rho, u, v, w, p)$, where $p$ is the pressure, and the transformation is

Received December 21, 1970.

AMS 1970 subject classifications. Primary 65M05, 65M10; Secondary 76L05.

Key words and phrases. Lax-Wendroff two-step method, three spatial dimensions, sufficient condition for linear stability, hydrodynamics.

Copyright $\odot 1972$, American Mathematical Society 


$$
\begin{aligned}
d W & =P \cdot d W^{\prime}, \\
A_{i} & =P A_{i}^{\prime} P^{-1},
\end{aligned}
$$

and so $M=P M^{\prime} P^{-1}$ and $G=P G^{\prime} P^{-1}$. This is done because the original $A_{i}$ are too complicated.

If we compute $P$ from (2.a), we get that

$$
\operatorname{det} P=\rho^{4} \partial e / \partial p \text {, }
$$

where $e$ is the internal energy per unit mass. It turns out that $P$ is triangular so that $\operatorname{det}\left(P^{-1}\right)=(\operatorname{det} P)^{-1}$.

Let $y_{i}$ be the normalized eigenvectors of $G$ (and $M$ ), then $\alpha_{i} P^{-1} y_{i}$ are the normalized eigenvectors of $G^{\prime}$ (and $M^{\prime}$ ), where $\alpha_{i}>0$ are the normalizing factors.

If we define

$$
\begin{aligned}
\Delta_{1} & =\left|\operatorname{det}\left(y_{1}, y_{2}, \cdots, y_{n}\right)\right|, \\
\Delta_{2} & =\left|\operatorname{det}\left(\alpha_{1} P^{-1} y_{1}, \alpha_{2} P^{-1} y_{2}, \cdots, \alpha_{n} P^{-1} y_{n}\right)\right|,
\end{aligned}
$$

and $\alpha=\alpha_{1} \cdot \alpha_{2} \cdot \cdots \cdot \alpha_{n}>0$, then

$$
\begin{aligned}
\Delta_{2} & =\left|\operatorname{det}\left[\alpha P^{-1}\left(y_{1}, y_{2}, \cdots, y_{n}\right)\right]\right| \\
& =\alpha\left|\operatorname{det} P^{-1}\right| \cdot\left|\operatorname{det}\left(y_{1}, y_{2}, \cdots, y_{n}\right)\right| \\
& =\alpha\left|\operatorname{det} P^{-1}\right| \Delta_{1}=\frac{\alpha}{\rho^{4} \partial e / \partial p} \Delta_{1} .
\end{aligned}
$$

This is the case because $\rho$ is always bounded away from zero and in the usual fluids the same holds for $\partial e / \partial p$ and consequently for $\operatorname{det} P^{-1}$. Therefore, $\Delta_{1}$ is bounded away from zero if and only if $\Delta_{2}$ is.

Rubin and Preiser found $M^{\prime}$ to be

$$
M^{\prime}=L\left[\begin{array}{ccccc}
u^{\prime} & \rho \operatorname{Cos} r & \rho \operatorname{Cos} s & \rho \operatorname{Cos} t & 0 \\
0 & u^{\prime} & 0 & 0 & 1 / \rho \operatorname{Cos} r \\
0 & 0 & u^{\prime} & 0 & 1 / \rho \operatorname{Cos} s \\
0 & 0 & 0 & u^{\prime} & 1 / \rho \operatorname{Cos} t \\
0 & \rho C^{2} \operatorname{Cos} r & \rho C^{2} \operatorname{Cos} s & \rho C^{2} \operatorname{Cos} t & u^{\prime}
\end{array}\right]
$$

where $L=\left(\operatorname{Sin}^{2} \xi_{1}+\operatorname{Sin}^{2} \xi_{2}+\operatorname{Sin}^{2} \xi_{3}\right)^{1 / 2}, \operatorname{Cos} r=\left(\operatorname{Sin} \xi_{1}\right) / L, \operatorname{Cos} s=\left(\operatorname{Sin} \xi_{2}\right) / L$, $\operatorname{Cos} t=\left(\operatorname{Sin} \xi_{3}\right) / L$ and $u^{\prime}=u \operatorname{Cos} r+v \operatorname{Cos} s+w \operatorname{Cos} t$.

A direct computation of its eigenvectors shows

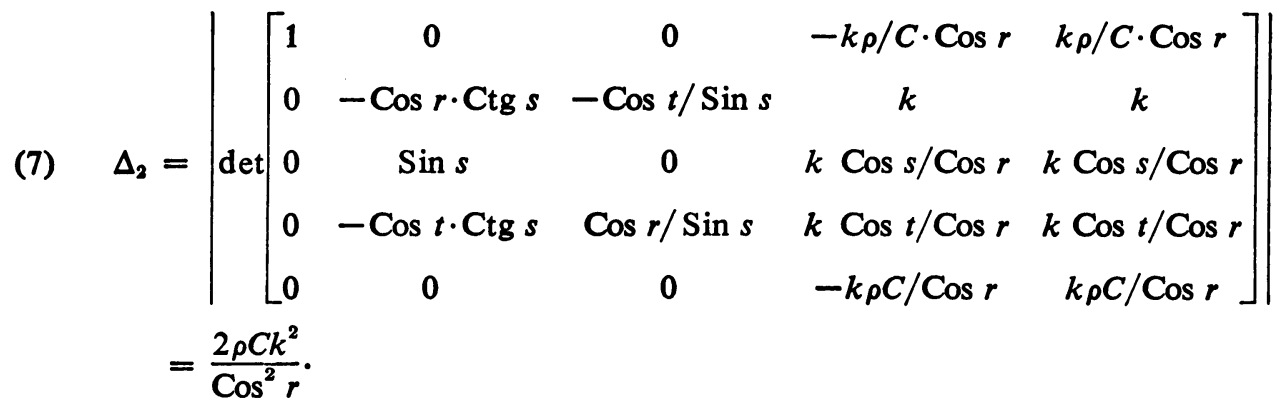


$k^{2}=\left(\operatorname{Cos}^{2} r\right) /\left(\rho^{2} C^{2}+1+\rho^{2} / C^{2}\right)$ is a normalizing factor so that finally

$$
\Delta_{2}=2 \rho C /\left(\rho^{2} C^{2}+1+\rho^{2} / C^{2}\right) .
$$

We see that $\Delta_{2}$, and so $\Delta_{1}$, is bounded away from zero. Hence, by the sufficiency theorem quoted above, the von Neumann condition, namely $\Delta t / \Delta x \leqq 1 / \sqrt{ } 3(|V|+C)$, implies linear stability.

Department of Mathematical Sciences

Tel-Aviv University

Tel-Aviv, Israel

1. E. L. RUBIN \& S. PREISER, "Three-dimensional second-order accurate difference schemes for discontinuous hydrodynamic flows," Math. Comp., v. 24, 1970, pp. 57-63.

2. R. D. Richtmyer \& K. W. Morton, Difference Methods for Initial-Value Problems, 2nd ed., Interscience Tracts in Pure and Appl. Math., no. 4, Interscience, New York, 1967. MR 36 \#3515.

3. H.-O. KREISS, "On difference approximations of the dissipative type for hyperbolic differential equations," Comm. Pure Appl. Math., v. 17, 1964, pp. 335-353. MR 29 \#4210.

4. S. Z. BuRSTEIN, "High order accurate difference methods in hydrodynamics," in Nonlinear Partial Differential Equations, W. F. Ames (Editor), Academic Press, New York, 1967, pp. 279-290. MR 36 \#510. 